\title{
Enfoque sistémico en \\ los procesos de \\ gestión humana
}

\section{César Nieto Licht ${ }^{*}$}

Systemic approach in

human resources

management processes

Approche systémique du processus de gestion des ressources humaines

Abordagem sistémica nos processos de Gestão Humana

"Candidato a doctor en Ciencias Empresariales de la Universidad Antonio de Nebrija-España. MBA de la Universidad Wollongong - Australia, Especialista en Gerencia de Recursos Humanos de la Universidad de los Andes - Colombia. Ingeniero Industrial de la Universidad de América - Colombia. Profesor Asociado de la Universidad EAN.

Gerente de Capacitación y Mejoramiento Continuo del Banco de Colombia.

Gerente de Desarrollo Humano del Banco Sudameris.

Director de Recursos Humanos de la Bolsa de Valores de Bogotá.

Gerente de Recursos Humanos de Geophysical Acquisition E Processing Services, GAPS. Director de Desarrollo Humano de la Universidad EAN. 


\section{RESUMEN}

Mediante la comprensión de la teoría sistémica, su evolución y sus aplicaciones se plantearán los retos generales y específicos que afrontan actualmente los profesionales de Gestión Humana para diseñar y desarrollar sus procesos de manera sistémica, generando valor para la organización y aportando significativamente al desarrollo de la empresa y de los seres humanos que la conforman. Por lo tanto, este artículo presentará una exploración por las herramientas de la era de la máquina, su impacto en el proceso de pensamiento de los administradores, el significado de la era sistémica y sus aplicaciones e incidencias en el diseño de los procesos. Finalmente, se presentarán los retos específicos que los profesionales de Gestión Humana deben afrontar para intervenir sus procesos bajo un enfoque sistémico.

\section{ABSTRACT}

Through the understanding of the systemic theory, its evolution and applications, general and specific challenges that Human Resources professionals face are described in this article for the design and development of systemic processes, creating added value to the organization and contributing to the development of the company and of the human resource that conforms it. Consequently, this paper shows the study of the tools of the machine era, its impact on the mental processes of managers, the real meaning of the systemic era and its implications in the design of processes. Finally, the specific current challenges that human resources professionals experience to intervene systemic focused processes are described in this article.

\section{RESUMÉÉ}

À travers la compréhension de la théorie systémique, de son évolution et de ses applications, nous analyserons les défis que doivent relever actuellement les professionnels du secteur des ressources humaines pour mettre en place des processus systémiques générant une valeur ajoutée à l'entreprise et améliorant sa croissance et la qualité du travail des employés. Ce document analyse les outils actuels de l'ère industrielle, leur impact sur le processus d'analyse des gestionnaires, la signification de l'ère systémique, ses implications et enjeux dans la conception des processus. L'article présentera enfin les défis particuliers auxquels font face les professionnels des ressources humaines qui utilisent les processus dans une approche systémique.

\section{RESUMO}

Através da compreensão da teoria sistémica, sua evolução e suas aplicações apresentarãose os desafios gerais e específicos que afrontam atualmente os profissionais em Gestão Humana para desenhar e desenvolver seus processos de maneira sistémica, gerando valor para a organização e contribuindo significativamente para o desenvolvimento da empresa $e$ as pessoas que fazem parte dela. Por conseguinte, este documento apresentará uma exploração das ferramentas da era da máquina, seu impacto no processo de pensamento dos administradores, o significado da era sistémica e suas aplicações e influência no desenho dos processos. Finalmente, apresentarão-se os desafios específicos que os profissionais em Gestão Humana devem afrontar para intervir seus processos com uma abordagem sistémica

\section{Palabras claves}

Teoría sistémica Valor organizacional

Gestión humana

Diseño de procesos Innovación

\section{Key words}

Systemic theory

Organizational added value

Human management

Process design

Innovation

\section{Mots clefs}

Théorie systémique

Valeur organisationnelle

Gestion des ressources humaines

Processus de conception Innovation

\section{Palavras-chave}

Teoria sistémica

Valor organizativo

Gestão humana

Desenho de processos

Inovação 


\section{INTRODUCCIÓN}

L a nueva lógica de la creación de valor y de la innovación está centrada en el desarrollo de los procesos de negocio de la organización. Si bien es necesario tener el conocimiento del saber cómo, es indispensable igualmente comprender el saber qué y el saber por qué del diseño de los procesos que generan valor en la organización. Esto implica que además de desarrollar el nivel de pensamiento en las categorías tradicionales de mejorar el hardware, el Software, el proceso de manufactura y servicios y la innovación en productos, es igualmente importante desarrollar un nuevo nivel de pensamiento que incluya elementos que permitan pensar los procesos del negocio de manera innovadora, diferente y sistémica. Por lo anterior, la innovación en los procesos del negocio se convierte en base fundamental para la generación de valor, ya que por medio ellos es que realmente se construirá a la innovación de la empresa, del cambio y de la cultura necesaria para afrontar los retos actuales de los negocios (Prahalad y Krishnan, 2008).

Hamel y Breen (2009) han resaltado la importancia de entender la innovación en el contexto actual, relacionándola en cuatro niveles que van desde la innovación operativa, la innovaciónen productosyservicios, lainnovación estratégica y finalmente la innovación administrativa, enfatizando que si bien todas son necesarias, cuando los problemas se hacen más complejos, realmente la innovación administrativa es única en cuanto a su capacidad para crear ventajas difíciles de copiar. Es así que este documento pretende presentar una propuesta de cómo, mediante procesos enmarcados en un pensamiento sistémico, se pueden repensar y rediseñar los procesos de Gestión Humana para que permitan aportar por sí mismos valores estratégicos a la organización.

\section{METODOLOGÍA DE LA INVESTIGACIÓN}

$\mathbf{P}$ ara alcanzar los objetivos planteados se procedió a investigar los referentes del tema sistémico, buscando encontrar las pautas generales del por qué las organizaciones se focalizan principalmente en hacer sus procesos eficientes y los desarrollan de manera no integrada ni articulada con el propósito final de la organización y de los mismos procesos. Esta afirmación recae en la forma como generalmente son vistos algunos de los procesos de Gestión Humana, que si bien buscan incrementar el aporte del trabajador y mejorar su compromiso, logran efectos totalmente contrarios como la aversión, el disgusto y la falta de valor agregado de procesos tan importantes como la evaluación del desempeño, la compensación, la selección y la capacitación, por nombrar algunos.

Así las cosas, se procedió a investigar algunos referentes de la teoría sistémica y los impactos que esta genera a nivel de estructura de pensamiento, entendiendo que esta última es la fuente básica que tienen los profesionales que diseñan los procesos de sus empresas. A partir de allí se buscó la forma de proponer -basándose en mejores prácticas y orientaciones conceptuales de algunos pensadores del tema de Gestión Humana- acciones concretas de intervención en la forma de pensar y de estructurar los procesos de Gestión Humana y por ende los retos de los profesionales del área.

Posteriormente se desarrolló el análisis de los procesos de Gestión Humana para proponerlos desde la perspectiva sistémica. Para el análisis de estos procesos se tuvieron en cuenta las mejores prácticas y los conceptos de los pensadores del tema que proponían esquemas sistémicos y relacionados con el tema de generación de valor enfocado al cliente y a los diferentes Stakeholders. 


\subsection{Primera fase metodológica}

\subsubsection{Perspectiva en la Era de la Máquina}

La primera forma de entender las organizaciones se puede relacionar con lo que Ackoff (2004) denomina la Era de la Máquina, explicando que esa forma de ver la vida y el trabajo, perduró por décadas y siglos en el pensamiento del ser humano. Su origen se basó en dos creencias que eran que el hombre había sido creado a imagen y semejanza de Dios y que, como se había creado así, era lo más cercano a Dios en el Universo; la segunda, que el hombre debería crear máquinas para que hicieran su trabajo. La revolución industrial fue una consecuencia de esta visión del mundo.

¿Pero a qué se referían con máquinas en esas generaciones? Eran objetos que podían usarse para aplicar energía a la materia, por lo cual las máquinas principales fueron la palanca, la polea, la rueda, el eje y el plano inclinado. Fue así como se hizo un esfuerzo en dividir el trabajo en tareas simples que algunas veces eran realizadas por seres humanos y otras por máquinas. Taylor, con su análisis del trabajo, fue el precursor de dividir los procesos en tareas, medirlas y estandarizarlas, e igualmente fue el que más influyó en la mecanización de las tareas. Así, personas y máquinas se sumaban para realizar un trabajo completo. El resultado de esto fue la producción industrializada y la línea de montaje que constituye la base central de la fábrica moderna.

Sin duda, los beneficios de la Revolución Industrial fueron muchos y significativos; sin embargo, es claro que la revolución industrial, en su esfuerzo por reemplazar al ser humano como fuente de energía, terminó deshumanizando el trabajo por cuanto lo redujo a simples tareas que eran lo suficientemente básicas para que las máquinas las llevaran a cabo, si no era de inmediato. La naturaleza y el lugar de trabajo se vieron impactados por este paradigma analítico que influyó en las generaciones que vinieron, incluso se puede decir que diseñaron el sistema de trabajo actual. $\mathrm{Si}$ hubiera otra forma de concebir el trabajo, muy seguramente hoy la naturaleza y el lugar de trabajo sería algo diferente a lo que se conoce

\subsubsection{El análisis. Primera herramienta de la Era de la Máquina}

El análisis de las cosas es la primera herramienta utilizada en la Era de la Máquina y consiste en separar las partes para inferir cómo funcionan y a partir de allí, extraer una comprensión del todo. Según Ackoff (2004) esta perspectiva tiene tres fases:

- Separar las partes que quieren entenderse.

- Comprender el comportamiento de las partes tomadas por separado.

- Reunir un entendimiento en una comprensión del todo.

Este método analítico influyó en la forma como se realizaban las investigaciones e impactó el proceso de análisis e investigación como sinónimos. Utilizar el método analítico lleva a la observación y experimentación que es una de las bases de la ciencia moderna.

\subsubsection{El reduccionismo: segunda herramienta de la Era de la Máquina}

El reduccionismo significa que toda realidad de las situaciones del mundo puede reducirse a elementos indivisibles fundamentales. Es así como desde la Era de la Máquina, para entender algo, era necesario separarlo conceptual o físicamente en sus partes, y continuando este proceso de separación se entendía cada una de ellas, incluso la más mínima partícula. En esa época se pensaba que todos los objetos poseían dos propiedades intrínsecas, denominadas masa y energía.

\subsubsection{El determinismo: tercera herramienta de la Era de la Máquina}

Posteriormente, según Ackoff (2004), al tratar de explicar el entendimiento de las cosas con la filosofía del reduccionismo (dividiendo el todo en sus partes hasta lograr su completa comprensión), inevitablemente se pasó a explicar todo como una relación entre las partes creyendo que todas las situaciones del mundo se podían explicar bajo el paradigma de causa-efecto, que por su uso común ha perdido el 
conocimiento popular con que fue creado. Se dice que una cosa es la causa de otra, su efecto, si la causa es tanto necesaria como suficiente. 'Necesaria' significa que para que ocurra un efecto es indispensable que exista esa causa. 'Suficiente' significa que si ocurre la causa, se asegura la ocurrencia del efecto. Ahora bien, en la Era de la Máquina se trabajó en el concepto de si todo tenía causa, el Universo también la tendría y la respuesta a cuál sería la causa se determinó como primaria y se atribuyó, por supuesto, a Dios.

La consideración importante que no se valoró en la Era de la Máquina fue el concepto del medio, que significa que las causas pueden llegar a los efecto, si y solo si, el medio donde se desarrolla es completamente idéntico. Esta situación no es normal cuando se habla de interacciones humanas por cuanto el medio no es igual y por consiguiente, el efecto tampoco lo será. Quizás este concepto se desarrolló así en la Era de la Máquina dado que los experimentos realizados se hacían en laboratorios aislados donde el medio siempre era idéntico y no afectaba la consecución del efecto.

\subsubsection{El mecanicismo: cuarta herramienta de la Era de la Máquina}

El mecanicismo marcó una época hasta nuestros días, ya que es la fusión de las doctrinas del reduccionismo y del determinismo. Se consideraba que el universo era una máquina, no solo semejante a ella, sino igual. Esta comparación es importante en el concepto de entender el mundo, ya que excluía frecuentemente un elemento importante en el determinismo que era el medio.

\subsubsection{La Revolución Industrial: quinta herramienta de la Era de la Máquina}

La Revolución Industrial significó la sustitución del hombre como fuente de trabajo por máquinas diseñadas y elaboradas por él mismo. La mecanización del trabajo hizo que el análisis de los procesos productivos se redujera a sus elementos más sencillos, que se hicieron a partir del entendimiento del proceso desagregado en partes elementales. Esta influencia tuvo un impacto significativo en el trabajo debido a que el esfuerzo por reemplazar al hombre con máquinas, redujo el trabajo a tareas elementales, diseñadas de manera simple para que las máquinas las realizaran, ya que eran repetitivas. La conclusión de esta práctica de pensamiento fue que el trabajo se deshumanizó (Ackoff, 2004).

\subsection{Segunda fase metodológica}

\subsubsection{Perspectiva de la administración sistémica}

Los modelos mentales diferenciadores se enmarcan bajo enfoques sistémicos que integran una nueva forma de ver los procesos y que presentan perspectivas integradoras para la solución de problemas y la estructuración de procesos de valor. Aunque la evolución de la perspectiva sistémica fue trazada desde la antigua Grecia, hasta después de la Segunda Guerra, evolucionó con fuerza en los negocios. Gharajedaghi (2005) muestra un excelente y profundo análisis sobre la evolución de los sistemas en el que explica cómo el proceso de pensamiento ha evolucionado de la Era Mecanicista a la Era de los Sistemas.

Ahora bien, tener pensamiento holístico no es suficiente para trabajar bajo un pensamiento sistémico. Forrester (1971) identificó varias características de un sistema complejo, las cuales se enuncian a continuación:

- Causa y efecto están siempre separados en espacio y tiempo.

- La solución de problemas que mejora una situación específica en el corto tiempo usualmente crea grandes problemas en el largo plazo.

- Como resultado de las dos primeras características, las personas usualmente fallan en aprender de sus errores.

- Los subsistemas y partes de un sistema interactúan usando múltiples canales y pasos no-lineales. Esta compleja interacción generalmente crea comportamientos que no son intuitivos. En consecuencia, lo que parece ser una decisión obvia termina siendo, de hecho, una mala decisión.

No es común que siempre los profesionales se concentren en las complejas interacciones de las situaciones. El concepto de causa-efecto es generalmente aprendido en la 
juventud por medio de simples situaciones que se entendieron con ejemplo más sencillos aun. Pero esa mirada simple no es como en realidad funciona la complejidad de las situaciones, ya que aunque se percibe, la realidad de las acciones como una serie de causas-efectos, las situaciones sociales y de interacción humana, son parte de un sistema mayor y al mirarse de manera simple se cometen errores en la solución. Utilizando las palabras de Senge en su clásico libro La quinta disciplina: "Los problemas de hoy derivan de las 'soluciones' del ayer" (1990, p. 90).

Alvin Tofler (1971) señala que los seres humanos están expuestos a lo que él llamó "choque del futuro", refiriéndose a que los humanos deben responder con rapidez y efectividad a las transformaciones que viven so pena de quedarse en parálisis y no responder efectivamente a su choque del futuro. Schon (1971) incluyó una segunda característica del cambio que es la "complejidad", afirmando que mientras más rápidas y complejas las variaciones del problema, más largo será el tiempo para solucionarlo y más corto será el tiempo de la vigencia que se encuentre para dicha solución.

Por la misma línea, Ackoff (2004) señala que aunque los cambios en general son inevitables, sus particularidades no lo son. Como solución para los cambios, el ser humano debe adaptarse rápidamente y aprender de la situación, y dado que no es posible controlar el cambio, es necesario responder a él y controlar las variables que lo impactan. La velocidad del cambio está inmersa en el medio y en los paradigmas mentales del ser humano y consiste en comprender el mundo y la concepción que se tenga de él

\subsubsection{La Era de los Sistemas}

Thomas Kuhn (1970) incluyó en su famoso libro "La estructura de la Revolución Científica" varios conceptos que explican la forma como se obtiene la información de parte de los investigadores, y afirmó que la objetividad

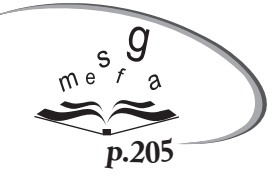
total en el proceso de investigación dependía mucho de la "subjetividad" del investigador, ya que este observaba lo que usualmente su paradigma le "decía" que observara. Este elemento de subjetividad lo incluyó como parte del proceso investigativo, y denominó "dilemas" a los problemas o preguntas que no podían resolverse o contestarse en un marco de la visión del mundo dominante y que por consiguiente se ponían en tela de juicio. Este marco dominante fue cuestionado con el estudio y posterior determinación del Principio de Incertidumbre propuesto por Heisenberg y aplicado a la física, cuando demostró que no podían determinarse simultáneamente dos propiedades fundamentales de una partícula atómica, porque cuando se aumenta la precisión en la determinación de una de ellas, disminuye la precisión de determinación de la otra. Esta demostración puso en tela de juicio que el mundo podía entenderse cabalmente, incluso en su creación. Esta teoría fortaleció la importancia de los dilemas y el entendimiento de que la visión mecanicista del mundo debería ampliarse (Bokulich, 2006). Es así como el proceso de investigación salió de los laboratorios para meterse en el "mundo real" y resolver los problemas de las organizaciones grandes y complejas. Igualmente, esta salida a la realidad hizo que los investigadores utilizaran en sus estudios campos interdisciplinarios del conocimiento que intensificaron la afectación de las causas y medios para buscar sus correspondientes efectos.

A finales de los años cincuenta existía consenso generalizado acerca de las similitudes interdisciplinarias y esto generó la preocupación compartida de los científicos en relación con el comportamiento de los sistemas. Esta situación puso de manifiesto que debía plantearse la modificación del mundo por medio de otro paradigma diferente a la Era de la Máquina, que iniciaba y se podía referir como la Era de los Sistemas (Ackoff, 2004).

Según Ackoff y Gharajedaghi (1996), un sistema es como el conjunto de dos o más elementos que satisfacen las siguientes condiciones:

- El comportamiento de cada elemento tiene efecto en el comportamiento del todo.

- El comportamiento de los elementos y sus efectos sobre el todo son interdependientes.

- De cualquier manera que se formen subgrupos de los elementos, cada uno tiene un efecto sobre el comportamiento del todo y ninguno tiene efecto independiente sobre él.

Con estas afirmaciones se entiende que un sistema no puede dividirse en sus partes, ya que cada pieza del sistema 
tiene sus propiedades - representadas en el resultado del sistema- que se pierden si se separan de este. De la misma forma, el sistema tiene unas propiedades que ninguna de las partes tiene. El ejemplo más claro es el cuerpo humano, que funciona como un sistema. Si se saca el sistema respiratorio, este no funciona por sí solo sin la interacción con otros. Igualmente pasa con el sistema completo, que no se puede representar solamente como el sistema respiratorio. Se concluye que cuando un sistema se separa de sus partes pierde sus propiedades esenciales, por lo tanto, un sistema es un todo y no puede entenderse por análisis.

\subsection{Pensamiento sistémico}

La síntesis de los elementos es la clave del pensamiento sistémico, del mismo modo que el análisis y la separación de los elementos hacían parte de la Era de la Máquina. Se puede afirmar que la síntesis y el análisis son procesos complementarios y por lo tanto no se puede afirmar que la síntesis caracterice a la Era de los Sistemas y el análisis a la Era de la Máquina, sino que ambos forman parte del proceso de pensamiento sistémico que los combina. El proceso de pensamiento en la Era de la Máquina tenía tres pasos indispensables:

- Descomposición de lo que va a explicarse.

- Explicación del comportamiento o propiedades del elemento.

- Agregación de estas explicaciones en una explicación del todo.

En el enfoque sistémico, de igual manera, se presentan tres pasos:

- Identificar un sistema del cual el objeto por explicar es una parte.

- Explicar el comportamiento o propiedades del sistema.

- Explicar el comportamiento o las propiedades del objeto en términos de su función o papel dentro del sistema.
Ackoff (2004) afirma que el pensamiento analítico intenta explicar un todo que debe separarse, mientras el enfoque sistémico intenta explicar una de las partes como perteneciente a un todo. El enfoque analítico reduce, mientras que el sistémico lo expande. Un ejemplo que explica esta diferencia es cómo se podría definir el término 'Universidad' bajo los dos enfoques. Un pensador de la Era de la Máquina explicaría el concepto diciendo que la Universidad se divide en facultades, después en departamentos, después en docentes, estudiantes y asignaturas; mientras un pensador sistémico miraría el proceso formativo (formación, investigación y extensión), sus objetivos y responsabilidades con respecto al sistema general y por último, explicaría la Universidad en términos de sus papeles o funciones dentro de la sociedad.

Estos enfoques no deberían producir resultados contradictorios pero se puede afirmar que el análisis se enfoca en la estructura y en cómo funcionan los elementos, mientras que la síntesis se enfoca en el propósito y en el por qué los elementos operan como lo hacen. El análisis mira hacia adentro de los elementos y la síntesis mira hacia afuera de ellos. Una de las diferencias que Ackoff (2004, p. 19) resalta es que "si cada parte del sistema, considerado por separado, se hace operar a la mayor eficiencia posible, el sistema como un todo no operará con la mayor efectividad posible", finalmente, afirma que "el desempeño de un sistema depende más de la manera en que interactúan sus partes que de la manera en que actúan independientemente cada una de ellas".

Otro elemento importante entre las partes y el todo se refiere a la necesidad de coordinar el comportamiento de las partes en el sistema. Sin embargo, Ackoff relaciona que siempre existe un conflicto por cuanto al separar las partes se busca la mayor efectividad de ellas, algunas veces pasando por alto la función de coordinación del sistema. El paradigma

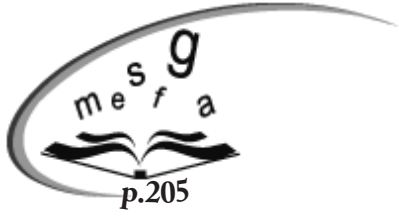
sobre el cual se trabaja este concepto, es que al mejorar las partes por separado, se puede mejorar el sistema. Sin embargo, el principio de los sistemas expuestos anteriormente afirma que no es posible. 


\subsubsection{Aplicaciones del pensamiento sistémico}

Ackoff (2004) propone las siguientes aplicaciones del pensamiento sistémico, denominándolas doctrinas del pensamiento sistémico, así:

\section{- Expansionismo}

En el pensamiento sistémico se piensa que puede comprenderse mejor el sistema si se expande la comprensión del mismo y no se reduce la comprensión a sus elementos constituyentes. Se afirma que la comprensión del sistema debe realizarse a través de incluir los suprasistemas que incluyen el sistema estudiado. Así las cosas, se podría pensar que el análisis de un sistema nunca tiene fin, por cuanto si se analiza el sistema o supra sistema que lo contiene, sería interminable el proceso. Igual situación se presentaría en la Era de la Máquina, que dividía al todo en sus partes y estas se dividían hasta el elemento más simple. Como resultado de estos procesos se ha llegado a la conclusión, según Ackoff (2004), de que podría acercarse a la comprensión total pero que el ideal nunca podrá ser alcanzado.

El expansionismo es importante por cuanto suministra una guía para resolver los problemas y al igual que en la Era Mecanicista, en la cual se dividía el todo en sus partes, el proceso de expansionismo de la Era de los Sistemas busca las soluciones desde afuera y solamente se abre camino al interior cuando allá (desde afuera) se fracasa.

\section{- Productor-producto}

Ackoff (2004), para explicar cómo el productor-producto reemplazó el concepto de causa-efecto, demostró que en las relaciones de causa-efecto no se tenía en cuenta el elemento medio que intervenía sustancialmente en lograr el efecto. Para explicar este concepto utilizó el ejemplo de un árbol que de ser plantado en el océano, en el ártico o en el desierto no produciría como consecuencia un árbol frondoso, ya que el medio no es propicio para su desarrollo. Contrariamente, si el árbol o la semilla fueran sembrados en un medio propicio, su desarrollo sería diferente, ya que es por este elemento que se convierte en árbol. Productor-producto, influenciado por el medio, es pues la forma como deben explicarse las cosas por encima de causa-efecto, ya que la ciencia basada en la relación productor-producto está llena de medio y no libre de él.

Por lo anterior, este concepto es fundamental ya que todo profesional debe incluir el medio como un elemento fundamental en sus procesos, especialmente en los de intervención humana como cultura y clima organizacional, por cuanto si no se trabaja en el medio es muy difícil que las causas por sí solas produzcan los efectos esperados.

\section{- Teleología ${ }^{1}$}

En el enfoque sistémico, la teleología se incluye por cuanto los fines (metas, objetivos e ideales) de un sistema podrían determinarse con tanta objetividad como el número de elementos que lo conforman. Aristóteles utilizó este concepto hace siglos pero lo definió de manera subjetiva, ya que para explicar el comportamiento humano utilizó la existencia de variables como las creencias, los sentimientos, las actitudes y los impulsos que no eran observables sino por quienes los experimentaban. Esta teleología se denominó teleología subjetiva.

Posteriormente se introdujo el concepto de la teleología objetiva por cuanto se afirmó que las creencias, los sentimientos, las actitudes y demás emociones son atribuibles a los seres humanos debido a que se reflejan en sus comportamientos y por lo tanto son observables. Estos conceptos no están detrás del comportamiento sino como propiedades observables del comportamiento de la persona. En otras palabras, la teleología aporta al enfoque sistémico debido a que estudia los resultados, no desde una perspectiva determinista $^{2}$ sino desde una perspectiva orientada a los elementos de entrada y a sus metas y fines (Macdorman y Sommerhof, 1994).

\footnotetext{
${ }^{1}$ Término que proviene del griego y que significa Telos (fin, meta o propósito) y logos (razón, explicación). De esta manera la teleología puede explicarse como la razón o explicación de algo en función de su fin o propósito.

${ }^{2}$ El determinismo explica que los actos que tienen los seres humanos no provienen de sus decisiones sino de los antecedentes que ha vivido. Es así como el determinismo genético relaciona que el comportamiento del ser humano viene dado por los genes familiares y si alguien es de mal genio, por ejemplo, se lo atribuye a los genes y no a su decisión de ser malgeniado.
} 
El enfoque teleológico significa, para los profesionales sistémicos, que deben centrarse en estos sistemas porque los mismos persiguen metas y son intencionados. Esto quiere decir que las acciones de los profesionales del área deben mirar perfectamente cuál es el propósito y alinear los elementos y el medio para que logren la intención final. Para relacionar el enfoque teleológico de la Era Sistémica con el de la Era Mecanicista, hay que anotar que anteriormente hasta el hombre era considerado como parte de un sistema; sin embargo, hoy en día se comprende la máquina únicamente con referencia a la finalidad para la cual es usada por el sistema. Las máquinas ordinarias sirven para finalidades de otros y no tienen objetivos propios, pero el ser humano, las organizaciones y las comunidades son sistemas con finalidades propias y así tienen que tratarse.

Este enfoque teleológico igualmente significa, que si bien algunas partes del sistema no tienen finalidades propias, la organización y el ser humano sí las tienen y debe entenderse su intervención de manera general y no particular. Un ejemplo son las áreas de una organización; si bien las áreas de finanzas, de mercadeo y de Gestión Humana no tiene finalidades propias, como partes de un sistema, sí las tienen. El profesional debe entender que si bien la división funcional del trabajo interrelaciona un conjunto de elementos en el cual cada uno tiene una función, no significa que él por sí solo sea igual al sistema. Por lo tanto, deberá coordinar sus partes para que finalmente la interacción del sistema genere los resultados esperados del sistema completo. Enfocándose en los procesos de Gestión Humana (léase selección, capacitación, evaluación de desempeño, compensación y bienestar, entre otros), estos son parte del sistema Organización y ninguno de ellos representa el sistema por sí solo, explicando que la coordinación de todos ellos debe enmarcarse dentro del sistema total y no solamente dentro de un sistema efectivo ${ }^{3}$ de Gestión Humana.

\section{- La revolución postindustrial}

Según Ackoff (2004), la revolución postindustrial desarrolló nuevos mecanismos que, causados por el avance de la ciencia, permitieron analizar y conocer otros

\footnotetext{
${ }^{3}$ Entiéndase como efectividad la suma de la eficiencia (adecuado uso de los recursos y procesos) y la eficacia (elaboración de productos que satisfacen las necesidades de los clientes).
}

elementos del sistema que en la Revolución Industrial no se consideraban. Estos elementos se desarrollaron básicamente por la dificultad de observar algunos fenómenos, como la electricidad o la corriente eléctrica, razón por la cual se desarrollaron instrumentos que permitieron hacerlos "observables", como es el caso del amperímetro, el ohmímetro y el voltímetro, entre otros, que permitieron entender mediante símbolos las propiedades de los objetos o eventos medidos. Estos instrumentos que emitían símbolos eran instrumentos de observación que representaban las propiedades de las máquinas pero no eran las máquinas en sí; sin embargo, hacían parte de ellas al reflejar su comportamiento y hacer más entendible sus efectos para el ser humano. Estas máquinas fueron las pioneras de la tecnología de la instrumentación, la cual es completamente diferente de la mecanización. A manera de analogía es lo que hacen los cuadros de mando integral, que no se hacen como propósitos finales, sino son instrumentos que sirven para entender el desempeño de las personas y de los sistemas en una organización.

La tecnología de la instrumentalización, de la observación y de la comunicación, cambió radicalmente el concepto mecanicista e introdujo a la humanidad en un concepto nuevo que posteriormente la Era de los Computadores fortaleció. Este nuevo concepto implicó que las máquinas pudieran controlar a otras máquinas y que se pudieran automatizar los procesos. Según Ackoff (2004), se pasó de la mecanización (reemplazo del músculo por parte de la máquina) a la automatización (reemplazo de la mente).

\subsubsection{Organizaciones sistémicas}

Otro reto que tiene el profesional de Gestión Humana es la necesidad de elaborar, diseñar e implementar una organización sistémica social que, según Grarajedaghi (1985, 1986) y Ackoff (1981, 1994), debe tener las siguientes características:

- Son organizaciones democráticas. El término democrático hace alusión a la capacidad de los miembros para tomar decisiones y actuar en consecuencia. El individuo puede ser influenciado por personas que tienen autoridad sobre él, pero este siempre decidirá actuar de acuerdo con sus criterios. 
- Ostentan una economía interna de mercado. La gestión por procesos ha arraigado este concepto que significa que en cada parte de la organización las áreas pueden comprar o vender su producción a cualquier cliente o proveedor y se regirán por criterios de calidad, precio y valor agregado.

- Poseen una estructura organizacional multidimensional. Se refieren a que las organizaciones deben estructurarse por la función que desempeñan (unidades cuyas salidas son de consumo interno), sus productos y servicios (usualmente los consumen los clientes externos a la organización) y sus usuarios (clientes que compran sus portafolios de productos y servicios).

- Utilizan un proceso de planeación interactiva. Significa que el proceso de planeación debe incluir, además de lo que se espera, los medios, el suministro de los recursos, la especificación de los pasos y el diseño del sistema de monitoreo y control tanto de la implementación como de los efectos del plan.

- Mantienen sistemas de apoyo de las decisiones. Significa implementar un sistema de retroalimentación que permita el registro de las expectativas generadas alrededor de cada decisión importante, los supuestos de información que lo sustentan y el proceso mediante el cual se llega a la decisión.

\subsection{Tercera fase metodológica}

\subsubsection{Pensamiento sistémico en los procesos de Gestión Humana}

Ulrich (1998) define que los profesionales de Gestión Humana deben trabajar principalmente en dos retos básicos para mejorar la efectividad de su proceso. Estos dos retos son: primero, mejorar los procesos internos del área y, segundo, repensar la creación de valor de los productos y servicios de Gestión Humana. Si estos dos retos son realizados adecuadamente, harán que los profesionales del área rediseñen sus productos y servicios y se conviertan en profesionales sistémicos que creen infraestructuras eficientes, tanto en los procesos internos como en la organización.

\subsubsection{Mejorar los procesos}

Consiste en eliminar, suprimir, simplificar, optimizar e innovar aquellas tareas de Gestión Humana que no están generando valor en el análisis del macrosistema y que usualmente se pueden realizar con inversiones de tecnología. Este mejoramiento de procesos debe darse en diferentes perspectivas y aplicaciones de la Era Sistémica, que se pueden definir de la siguiente manera según Ulrich, Yeung y Brockbank (2007).

\subsubsection{Atender las necesidades estratégicas de la empresa}

La primera aplicación de la era sistémica es el expansionismo, que significa comprender el proceso en el macrosistema superior. Pues bien, Pfeffer (1994) enfatiza que los gerentes generales han cambiado sus expectativas con respecto a los resultados de Gestión Humana. Es así como la alta dirección tiene expectativas de que dicha área sea un gestor del cambio, desarrolle la ventaja competitiva por medio de la gente y optimice el proceso de implementar la estrategia. Este es un reto significativo para el equipo de Gestión Humana; no en vano Ulrich, et al. (2007) afirman que se trabaja para que una de las competencias de los profesionales sea la de aliado del negocio.

Con el propósito de enfocarse en cumplir las necesidades estratégicas, el profesional de Gestión Humana debe entender, principalmente, su responsabilidad de lograr la estrategia organizacional y determinar el impacto de los procesos que implementará al interior de su área.

Ahora bien, el sistema tradicional de medición estratégica para las empresas era el sistema contable, ya que había un énfasis importante en el corto plazo y poca disposición para invertir en el largo plazo y por ende, en la creación de valor de la empresa. A partir del auge de la creación de valor en el largo plazo (activos intangibles e intelectuales) que generan un crecimiento futuro como, por ejemplo, mejora de procesos, desarrollo de los recursos humanos, tecnologías de la información, bases de datos y sistemas, la estrategia tomada por una empresa empieza a adquirir mayor valor y se convierte en su sostenibilidad o su terminación (Wright, Gardner, Moynihan, y Allen, 2005). 
López y Nevado (2008) evalúan dos opciones para determinar el impacto estratégico de las empresas: el Cuadro de Mando Integral de Kaplan y Norton y el Modelo de Análisis Integral que permite medir y gestionar los capitales intangibles responsables fundamentales del crecimiento de las organizaciones y de los países. Este modelo, según los autores, aporta ventajas sobre el cuadro de mando integral, ya que explica cómo elaborar los indicadores con un sistema de alerta dinámico que proyecta o simula actuaciones hacia el futuro, planteando su adecuación y controlando sus beneficios. Adicionalmente, proporciona una cuantificación del valor de los capitales intangibles y los correlaciona con la estrategia, indicando cuáles hay que reforzar y dónde evitar que se sobredimensionen.

Como conclusión de la atención a las necesidades estratégicas se debe afirmar que es necesario contar con un modelo de evaluación sistémica que incluya las necesidades de la organización y el aporte de cada perspectiva a este fin.

\subsubsection{Satisfacción del cliente}

La siguiente aplicación de la era sistémica es la de productoproducto, que para este caso está influenciada por la filosofía de la calidad total en las funciones de Gestión Humana. El propósito de esta función se basa en reorganizar los procesos para suministrar mejor respuesta, generar valor y entregar servicios efectivos. Sin embargo, el área de Gestión Humana es percibida en las empresas más como un obstáculo que como un facilitador y, peor aun, al copiar los programas de otras compañías, es percibida como suministrador de programas que no están diseñados a la medida, sino que son elaborados de manera descontextualizada y desalineada (Yeung y Brockbank, 1995).

El reto en la satisfacción del cliente consiste en valorar el nivel de cumplimiento de las expectativas de los clientes. $\mathrm{Al}$ respecto existen varias metodologías como la norma de calidad ISO, el modelo europeo de la excelencia, el modelo SERVQUAL-SERVPERF y la metodología WIGN (Walker Information Global Network), entre otros.
El sistema ISO recalca la satisfacción de los clientes en sus principios, que son: organización enfocada al cliente, enfoque a procesos, enfoque del sistema hacia la organización, liderazgo, participación del personal, enfoque objetivo hacia la toma de decisiones, mejora continua, relación beneficiosa con el proveedor. Igualmente, el Modelo Europeo de Excelencia (EFQM) relaciona dentro de sus criterios: liderazgo, planificación y estrategia; personas, colaboradores y recursos; procesos, resultados en los clientes, resultados en las personas, resultados en la sociedad, rendimiento final de la organización.

Por su parte el modelo SERVQUAL - SERVPERF mide los elementos intangibles como: apariencia de las instalaciones físicas, equipos empleados y materiales de comunicación, fiabilidad (habilidad de prestar el servicio prometido de forma precisa), capacidad de respuesta (deseo de ayudar a los clientes y servirles de forma rápida), seguridad (conocimiento del servicio prestado y cortesía de los empleados así como su habilidad para transmitir confianza al cliente), empatía (atención individualizada al cliente). Por su parte, la metodología WIGN evalúa el desempeño organizacional por parte de los usuarios, los productos y los servicios, valorando la lealtad, la satisfacción, la fidelidad y el cumplimiento de las expectativas de los clientes.

El aporte significativo que gestión humana puede dar a esta valoración de la calidad y la entrega de los servicios a sus clientes internos y externos, es sin duda, el diseño e implementación de evaluación del desempeño. Si bien pocas empresas aplican el proceso de evaluación del cliente y esos resultados son transferidos directamente a la evaluación de los trabajadores, esa tendencia ya se implementó en el conocimiento de los profesionales de gestión humana y actualmente es llevada a la práctica gracias a la poderosa ayuda de los sistemas tecnológicos que permiten parametrizar el proceso, para que la acción sea automática desde el momento que el cliente evalúa el servicio requerido. Igualmente, la implementación de los procesos de calidad asegura que bajo la metodología de proveedorentrada-proceso-entregable-cliente, se diseñen los procesos enfocados en la satisfacción del cliente (Wick, Pollock, y Jefferson, 2010). 


\subsection{Volver los procesos eficientes}

\subsubsection{Reducir costos}

La aplicación de la Era Sistémica denominada revolución postindustrial, se utiliza en una de las prioridades de las compañías en momentos de crisis, que es reducir sus costos, y uno de los ítems, que por lo general se escoge, es reducir costos en la línea de gerencia y en la línea del Staff. Este efecto ha impactado el área de Gestión Humana, pues estos departamentos se han reducido en forma considerable, como lo demuestra Cameron (1994) al afirmar que el número de personas de Gestión Humana en compañías como Hewelt Packard e Intel iniciaron una reducción del Staff del área disminuyendo la relación de personas del área vs. el número total de empleados de 1/45 a 1/100, con el fin de preparar la organización y de exigir al área prestar unos nuevos servicios que generen mayor valor. Pero la reducción de costos no solo se ha centrado en el descenso de número de personas, también las empresas se han centrado en racionalizar sus operaciones y sacar provecho de las economías de escala reduciendo operaciones duplicadas y siendo más eficiente en el uso de los recursos (Butteriss, 2001).

\subsubsection{Aplicar la tecnología}

Utilizando la misma aplicación de la Era Sistémica del ítem anterior, denominada revolución postindustrial, Hamer y Champy (1994) enfatizaron que la tecnología ayudaba a las organizaciones a mejorar el desempeño de sus procesos por medio de intervenir y mejorar los procesos, con lo cual podrían hacerse mejoras radicales al desempeño de los mismos si se sustituía la rutina transaccional de las actividades por poderosos sistemas de información. Igualmente, al simplificar los procesos, dando acceso a la información a los empleados y empoderándolos a que tomaran decisiones basados en sistemas inteligentes, se podría mejorar el desempeño organizacional. Reingeniería no era solamente basarse en tecnología, ya que bien lo diferenciaban de la automatización, sino más bien se trataba de rediseñar los procesos bajo un nuevo enfoque sistémico y más simple.

\subsection{Crear valor para la organización}

Esto es repensar la creación de valor de Gestión Humana por medio de algunas acciones que permitan que el impacto pueda medirse y pueda generarse visiblemente bajo la medición de resultados.

\subsubsection{Reenfocar los propósitos del área}

La aplicación de la Era Sistémica utilizada en este reto es la teleología. Fitz-enz (2000) presenta un modelo de Gestión Humana cuyo centro o corazón es el proceso de evaluación de desempeño. A partir de ahí despliega el diseño de otros procesos como la planeación, adquisición, soporte, desarrollo y retención de los colaboradores. Así las cosas, propone que todos los demás procesos partan del diagnóstico que suministra la evaluación de desempeño para que de allí se planteen las brechas de desempeño y se ajusten los otros procesos del área. La evaluación que diseñe Gestión Humana debe procurar no sólo medir los resultados, sino también los comportamientos de los trabajadores. Para este

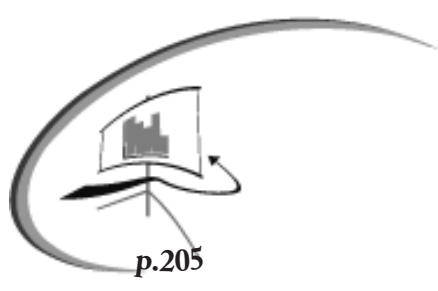
fin deben incluirse en los modelos de competencias los comportamientos esperados y sus respectivos indicadores de medición. Es importante que los fines no justifiquen los medios, ya que la creación de cultura es fundamental en el proceso de desarrollo organizacional y que la evaluación cumpla sus propósitos estratégicos.

Igualmente, la evaluación debe suministrar una retroalimentación más estricta del cliente interno y externo por cuanto, si bien las empresas han mejorado en la evaluación jefe-colaborador, falta camino por recorrer en cuanto a la valoración de otros puntos de vista que aprecien si las expectativas se están cumpliendo satisfactoriamente y si los clientes están satisfechos o inconformes, no solo con la persona (competencias, actitud y disposición) sino también con el producto.

La planeación desarrolla un papel fundamental en la Gestión Humana y fue ampliamente practicada hasta mediados de los ochenta. Durante los noventa el sentido de urgencia 
de las empresas primó sobre esta función haciendo que lo operativo y las necesidades del negocio tuvieran prioridad sobre esta responsabilidad del área. Fitz-enz define que la planeación de las capacidades de la empresa está tomando más fuerza actualmente, ya que se hace necesario que las funciones de gestión humana se integren con las operaciones de la empresa y se atiendan los requerimientos de colaboradores talentosos de la manera más rápida posible. Pero planear no es solo mirar las necesidades internas de la organización, pues requiere adicionalmente conocer, analizar y plantear soluciones acerca de lo que sucede con la fuerza laboral y con el entorno que afecta a los colaboradores y a los potenciales candidatos a ingresar a la compañía.

El proceso de adquisición de talento se está convirtiendo en punto neurálgico de la gestión humana debido a que el modelo tradicional de contratación permanente ha cambiado por un modelo de contratos a corto plazo y aún se hace mediante una firma intermediaria o temporal. De igual forma, la explosión de contratación por Outsourcing hace que las empresas busquen formas innovadoras de buscar el talento necesario. Pero la incidencia más compleja en la adquisición de talento se está dando por el cambio de paradigma mental de muchos trabajadores que ya no solo buscan contratarse por largos periodos de tiempo, sino que buscan mejorar su calidad de vida. El proceso de atracción se ha transformado de un proceso pasivo, donde las empresas esperaban por los candidatos, a uno activo, donde las empresas buscan el talento (Jerico, 2008).

La retribución participa con un papel fundamental en los resultados de gestión humana por cuanto ya no es suficiente pagar salarios justos y proveer un razonable nivel de seguridad para mantener a los trabajadores talentosos. Numerosas estrategias de retribución están tomando lugar en las empresas y estas no solo están centradas en la tradicional equidad interna y competitividad externa, sino que cada vez es más frecuente que las organizaciones desarrollen paquetes de compensación que incluyen la compensación flexible $^{4}$, la compensación variable ${ }^{5}$, la compensación basada en el desempeño, el salario emocional ${ }^{6}$, demás estrategias fundamentales para mejorar el nivel de vida del empleado (Holbeche, 2004).

El desarrollo de los colaboradores enfocado en programas de capacitación ha sido una de las responsabilidades en las que se han invertido más recursos. Probablemente el área de desarrollo es la responsabilidad que más ha centrado su atención los profesionales de Gestión Humana en las últimas dos décadas. Fitz-enz (2000, p.114) relaciona que la industria de los Estados Unidos invierte alrededor de 75 billones de dólares por año en desarrollo de los empleados, según la revista "Training Magazine". Esta suma es considerable y quizás es el tema de mayor debate en la determinación del ROI de los recursos invertidos en Gestión Humana.

Dos objetivos estratégicos hacen parte de los retos del área y tienen que ver, en primera instancia, con enfocar no solamente el mejoramiento de las competencias técnicas sino también el desarrollo de las competencias personales o del carácter. El primer objetivo, es la inteligencia emocional, la aplicación de los principios y valores corporativos y el desarrollo de comportamientos no tóxicos ${ }^{7}$. El segundo objetivo estratégico, tiene que ver con la focalización de la inversión de los programas de capacitación en programas que promuevan el desarrollo de las competencias estratégicas. Bersin (2004) desarrolló una matriz de inversión de los recursos de capacitación, la cual enfatiza que el porcentaje mayor de la inversión debe hacerse en desarrollar las competencias estratégicas y permitir por ende que la empresa gane mercado. Por supuesto, también deben invertirse recursos en programas que aseguren la operatividad de la empresa, pero este porcentaje de inversión debe ser menor.

\footnotetext{
${ }^{4}$ Estrategia salarial utilizada para generar mayor ingreso neto, sin modificar el salario básico. En Colombia esto es permitido por la Ley y algunas formas de utilizarla es mediante vales para la alimentación.

${ }^{5}$ Estrategia de compensación que está en relación directa con el desempeño superior. En Colombia es utilizada frecuentemente en el área comercial, donde se asigna un salario básico fijo y un salario variable que se paga de acuerdo al volumen de ventas.

${ }^{6}$ Salario que se entrega en beneficios no monetarios.

${ }^{7}$ Bernardo Stamateas escribió el libro Gente Toxica, donde explica los comportamientos de personas que sin atacar directamente a los que le rodean, van minando su autoconfianza y los hace sentir culpables, mediocres, malos, entre otros. Tóxico significa que no lo mata de repente, pero que lo va matando poco a poco.
} 
La retención de los trabajadores es el último paso en el modelo de gerencia de capital humano y se relaciona con el reto de los profesionales de gestión humana de mejorar la relación con los empleados y buscar que los más talentosos permanezcan en la organización. Fitz-enz (2000) relaciona que la retención contribuye a reducir los costos de adquisición, de entrenamiento, de supervisión y que el mantener los talentos mejora la calidad, la innovación y el servicio de la compañía. Si este reto se mira desde esta perspectiva, el objetivo es que los talentos permanezcan en la organización y se reinventen cíclicamente en ella.

Este reto ha hecho que programas de ambiente laboral se trabajen intensamente en las organizaciones con el fin de disminuir en alguna medida las causas de salida de los colaboradores. Algunas de las razones por las cuales los empleados dejan las organizaciones, se presentan cuando el trabajo o la empresa no satisface las expectativas, cuando el trabajo no encaja en el proyecto de vida de los colaboradores, se recibe poca o ninguna retroalimentación, hay posibilidad de poco desarrollo o crecimiento, se percibe mucha desorganización e injusticia en la toma de decisiones, el trabajo no permite un balance entre la vida personal y la profesional y finalmente, cuando hay poca confianza en los jefes.

\subsubsection{Convertirse en proveedor de servicios de consultoría}

La aplicación de la Era Sistémica utilizada en este reto es la productor-producto. Retomando el punto anterior y dada la experiencia de los profesionales de gestión humana, el área se convertiría en un asesor de productividad bajo ambientes sanos de trabajo. Butteris (2001) recalca que se debe rediseñar la función corporativa para convertirla en una consultoría de la dirección de empresas sobre evaluación, planeación, adquisición, desarrollo, retribución y retención del talento en desarrollo de los activos humanos de la organización.

\subsubsection{Manejar a gestión humana como un centro de costos independiente}

Utilizando la aplicación de la Era Sistémica denominada revolución postindustrial, Yeung, Brockbank y Ulrich
(1994) proponen que una estrategia para mejorar la calidad de la contribución de gestión humana es convertirla en un centro de costo donde tenga ingresos, egresos, "facture" por sus productos y servicios y permita a sus clientes que contraten con ellos o con terceros externos. Permitir esta figura en la organización es dejar que las áreas de servicios trabajen como monopolios y requiere de una conciencia alta en cuanto a los estándares mínimos que deben cumplir los competidores y los mismos recursos humanos a nivel interno. Sin embargo, resulta novedoso que el desempeño se vea compensado por la opinión de un cliente que califica bien o mal el aporte realizado por su centro de costo independiente.

\subsubsection{Redefinir las responsabilidades de los gerentes de línea}

La aplicación de la Era Sistémica utilizada en este reto es la teleología, y así lo evidencia Hewlett Packard cuando fue uno de los pioneros en los años cincuenta, en designar el manejo del personal a los gerentes de línea. Sin embargo, desde el año 1956 hasta finales de los ochenta, esta responsabilidad se trasladó al área de personal o más recientemente denominada gestión humana. Esta propuesta, presentada por Yeung y Broackbank (1995), sugiere que la redefinición de las responsabilidades de los gerentes de línea podrían mejorar los objetivos sugeridos en la perspectiva uno, que son los de reducir costos, orientarse a las necesidades de los clientes y principalmente enfocarse en los aspectos estratégicos: fortaleza de esos mismos gerentes. En estos casos gestión humana ocuparía un papel de consultor, que es uno de los siete retos planteados por Butteris (2001) en el que se espera del área que transforme su función en una responsabilidad más consultiva que administrativa.

\subsubsection{Implementar prácticas de alto desempeño ajustadas a la organización}

Utilizando la aplicación de la Era Sistémica denominada expansionismo, Huselid (2005) y Subramony (2006) demuestran el impacto positivo que tiene la utilización de las mejores prácticas en el desempeño empresarial. Aunque las diferentes prácticas varían de empresa en empresa, lo que sí es común a todas es que generalmente se centran en contar 
con colaboradores competentes, motivados y desarrollados, y con procesos rigurosos de selección, recompensas por el desempeño, entrenamiento y compromiso. Estos estudios demuestran que las empresas que desarrollan las mejores prácticas han realizado inversiones significativas en alinear sus procesos de gestión humana que reflejen claramente su enfoque al alto desempeño. Adicionalmente, estos esfuerzos han conseguido que su éxito se convierta en una herramienta difícil de imitar, convirtiéndose en una ventaja competitiva. Los estudios también han confirmado que todas las mejores prácticas no aplican en todas las organizaciones por cuanto es necesario que estas sean ajustadas a la empresa (Pfeffer y Sutton, 2000). Igualmente, se ha confirmado que es necesario entender el medio en el que se desarrolla la práctica, puesto que este es un elemento, como lo menciona Ackoff anteriormente fundamental en el resultado de la práctica (Datta, Gutthrie y Wright, 2005, p. 48).

\section{CONCLUSIONES}

Ce evidencia la necesidad de comprender los procesos $\checkmark$ de gestión humana bajo una perspectiva sistémica, ya que bajo esta deben aplicarse el diseño y la implementación de los procesos del área. Comprender las herramientas de la Era de la Máquina y las aplicaciones de la Era Sistémica permitirá a los profesionales que diseñen sus procesos impactar más a la organización, encontrar el sentido original del por qué de los procesos e incrementar el sentido de pertenencia y de productividad de los trabajadores. Estas aplicaciones sistémicas permitirán que los procedimientos del área se estructuren para mejorar los procesos y hacer que estos generen mayor valor a la organización mediante los retos específicos planteados en este documento.

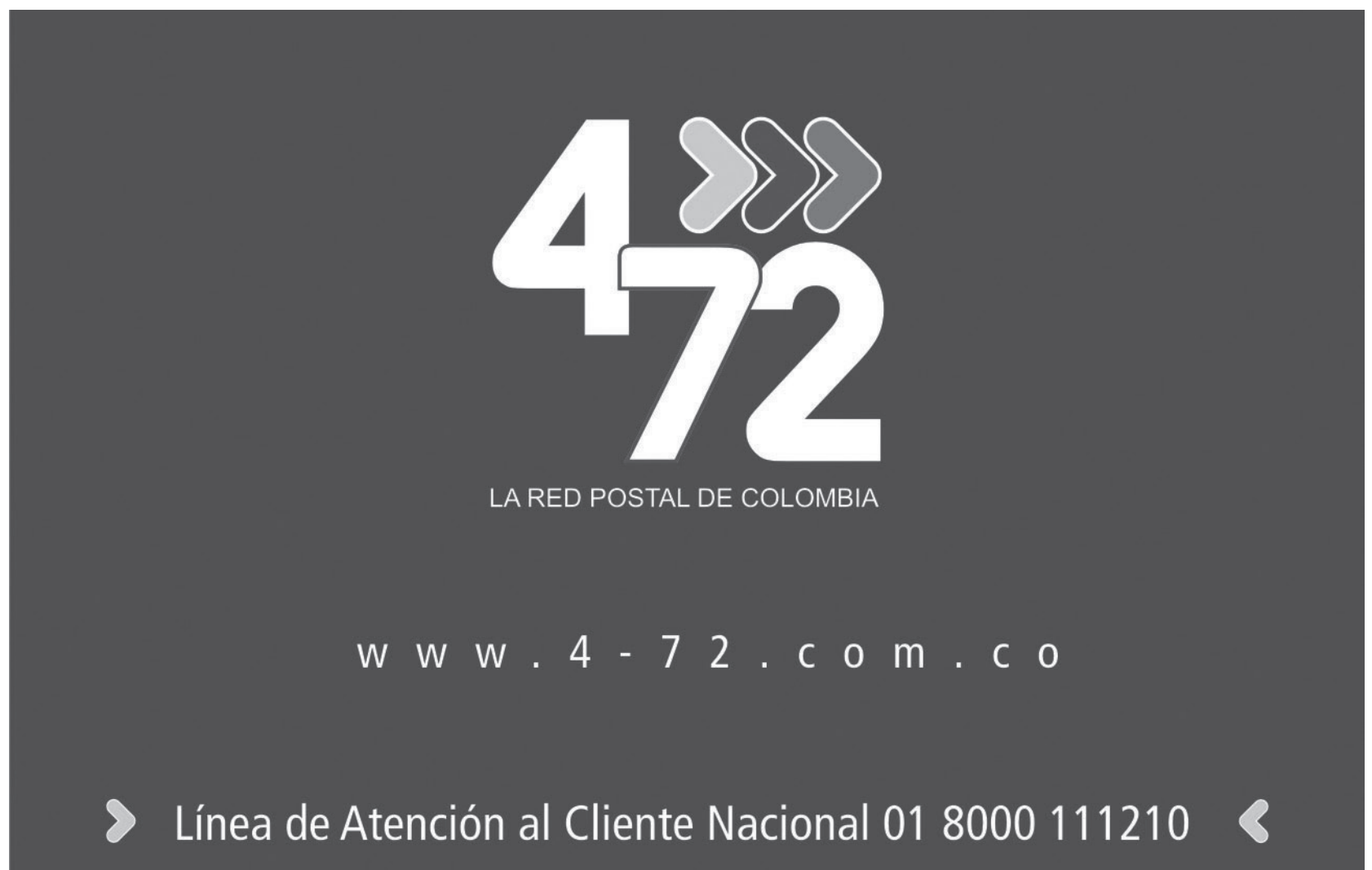




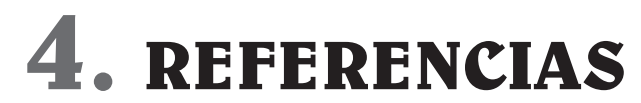

Ackoff, R. (1981). Creating the corporate future. New York: Wiley.

Ackoff, R. (1994). The democratic corporation. Oxford: Oxford University Press.

Ackoff, R. (2004). El paradigma de Ackoff. México: Limusa.

Ackoff, R. y Gharajedaghi, J. (1996). Reflections on systems and their models. Systems Research, 13-23.

Bersin, J. (2004). The blended learning. New York: Pfeiffer Press.

Bokulich, A. (2006). Heissenberg meet Kuhn: closed theories and paradigms. Philosophy of Science, 73, 90-107.

Butteriss, M. (2001). Reinventado Recursos Humanos. Barcelona: Gestión 2000.

Cameron, K. (1994). Strategies for successful organizational downsizing. Human Resources Management, 33, 189-211.

Datta, D., Gutthrie, J. y Wright, P. (2005). Human resource management and labor productivity: does industry matter? Academy of Mangement Journal, 135-145.

Fine, C. (2000). Clockspeed - based strategies for supply chain management. Production and Operation Management, 213221.

Fitz-Enz, J. (2000). The ROI of human capital: measuring the economic value of employee performance. New York: Amacon.

Forrester, J. (1971). The counterintuitive behavior of social system. Technology Management, 73(3), 52-68.

Gharajedaghi, J. (2005). System thinking: managing chaos and complexity. Worbun: Butterworth-Heinemann.

Hamel, G. y Breen, B. (2009). El futuro de la administración. Buenos Aires: Norma.

Hamer, M. y Champy, J. (1994). Reingenería. Bogotá: Norma.

Holbeche, L. (2004). Aligning Human Resorces and Business Strategy. 2nd Ed. Oxford: BH.

Huselid, M. (2005). The impact of human resources management practices on turnover, productivity and corporate financial performance. Academy of Management Journal, 38, 635-673.

Jerico, P. (2008). La nueva gestión del talento. Madrid: Prentince Hall.

Kuhn, T. (1970). The structure of scientific revolution. Chicago: University of Chicago Press. 
Lopez, V. y Nevado, D. (2008). Análisis integral como sistema de medición y gestión de capitales intangibles en organizaciones y territorios. Economía aplicada, 26, 119-137.

Macdorman, K. y Sommerhof, G. (1994). An account of consciousness in physical and functional terms. Integrative Physioligical and Behavioarl Science, 29, 38-46.

Pfeffer, J. (1994). Competitive advantage through people. Boston: Hardvard Business School Press.

Pfeffer, J. y Sutton, R. (2000). The knowing-doing gap: how smart companies turn knowledgee into action. Boston: Harvard Business School Press.

Prahalad, C. y Krishnan, M. (2008). The new age of innovation. New York: Mc Graw Hill.

Schon, D. (1971). Beyond the stable state. New York: Ramdom House.

Senge, P. (1990). La quinta disciplina. Madrid: Granica.

Subramony, M. (2006). Why organization adopt some human resource mangement practices and reject others: an explorations of rationales. Human Resources Management, 45, 195-210.

Tofler, A. (1971). Future Schok. New York: Batam Books.

Ulrich, D. (1998). Recursos humanos champions. Buenos Aires: Granica.

Ulrich, D., Brockbank, W., Johnson, D., Sandholtz, K. y Younger, J. (2007). Competencias de recursos humanos. Bogotá: Acrip.

Wick, C., Pollock, R. y Jefferson, A. (2010). The six disciplines of breakthrough learning.2nd Ed. San Francisco: Pfeiffer.

Wright, P., Gardner, T., Moynihan, L. y Allen, M. (2005). The relationship between HR practices and firm performance: examining casual order. Personnel Psychology, 58, 409-446.

Yeung, A. y Brockbank, W. (1995). Reengineering HR through information technology. Human Resources Planning, 18, 2437.

Yeung, A., Brockbank, W. y Ulrich, D. (1994). Lower cost, higher value: Human resources function in transformation. Human Resources Planing, 17, 1-16.

Zeleny, D. (2001). Autopoiesis (self-production) in SME nerworks. Human System Management, 20, 201-207. 


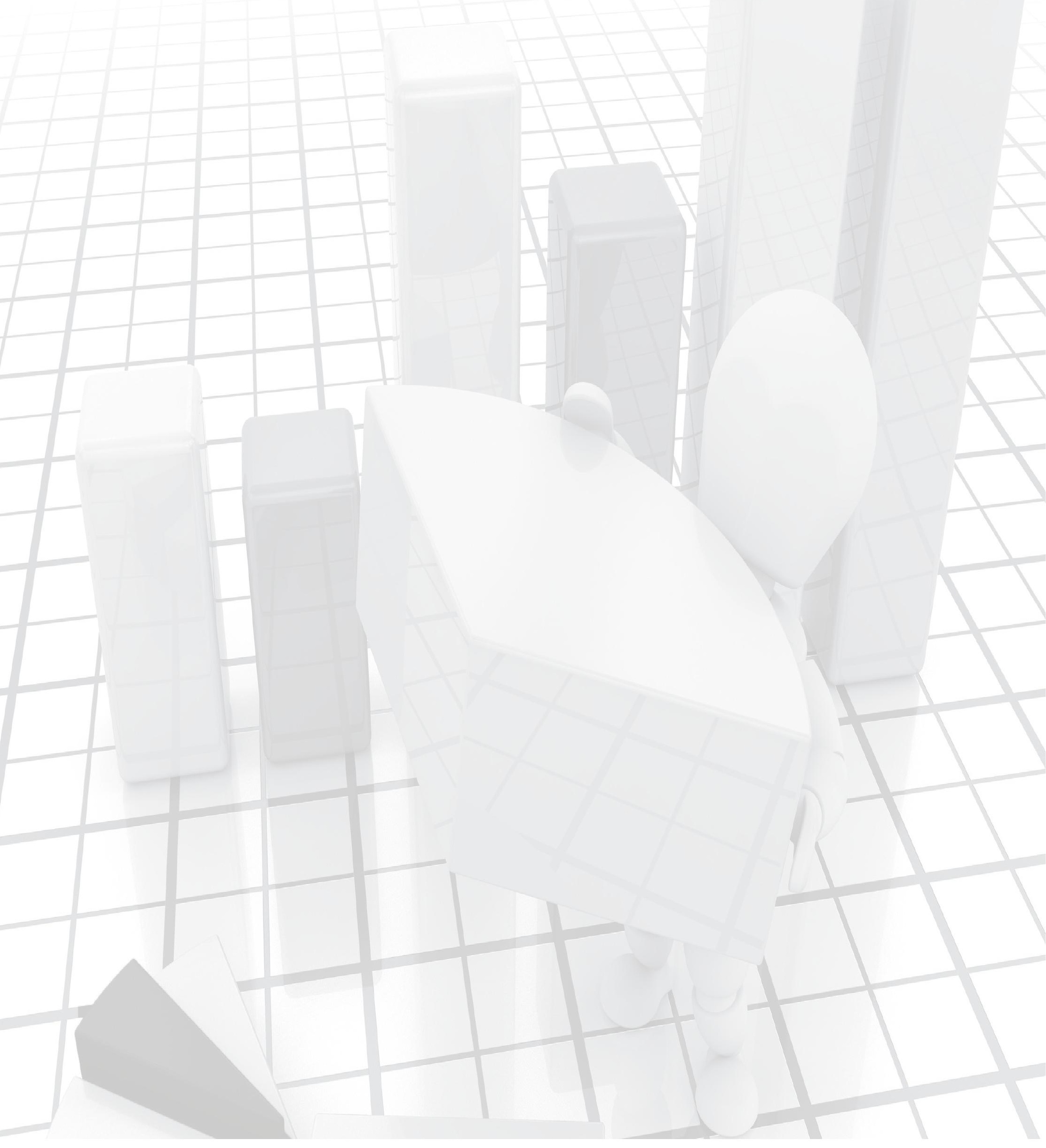

